\title{
Therapeutic Strategies in Acute Intracerebral Hemorrhage
}

\author{
H. Bart Brouwers • Joshua N. Goldstein
}

Published online: 4 December 2011

(C) The American Society for Experimental NeuroTherapeutics, Inc. 2011

\begin{abstract}
Intracerebral hemorrhage is a devastating disease, and no specific therapy has been proven to reduce mortality in a randomized controlled trial. However, management in a neuroscience intensive care unit does appear to improve outcomes, suggesting that many available therapies do in fact provide benefit. In the acute phase of intracerebral hemorrhage care, strategies aimed at minimizing ongoing bleeding include reversal of anticoagulation and modest blood pressure reduction. In addition, the monitoring and regulation of glucose levels, temperature, and, in selected cases, intracranial pressure are recommended by many groups. Selected patients may benefit from hematoma evacuation or external ventricular drainage. Ongoing clinical trials are examining aggressive blood pressure management, hemostatic therapy, platelet transfusion, stereotactic hematoma evacuation, and intra-
\end{abstract}

H. B. Brouwers · J. N. Goldstein

Division of Neurocritical Care and Emergency Neurology, Department of Neurology, Massachusetts General Hospital, Harvard Medical School,

Boston, MA 02114, USA

H. B. Brouwers

Center for Human Genetic Research,

Massachusetts General Hospital, Harvard Medical School,

Boston, MA 02114, USA

J. N. Goldstein

Department of Emergency Medicine,

Massachusetts General Hospital, Harvard Medical School,

Boston, MA 02114, USA

H. B. Brouwers $(\square)$

J. Philip Kistler Stroke Research Center,

Massachusetts General Hospital,

175 Cambridge Street - Suite 300,

Boston, MA 02114, USA

e-mail: brouwers@chgr.mgh.harvard.edu ventricular thrombolysis. Finally, preventing recurrence of intracerebral hemorrhage is of pivotal importance, and tight blood pressure management is paramount.

Keywords Intracerebral hemorrhage - Diagnosis .

Treatment $\cdot$ Blood pressure $\cdot$ Anticoagulation $\cdot$ Surgery.

\section{Introduction}

Incidence

Spontaneous intracerebral hemorrhage (ICH) accounts for 10 to $15 \%$ of all strokes worldwide or 10 to 30 cases per 100,000 people per year [1]. Patients with ICH show the worst outcome of all stroke subtypes with a 30 day mortality rate of 30 to $50 \%$ [2-5]. Moreover, longterm outcomes of ICH are even more devastating, with $75 \%$ of patients severely disabled or deceased at 1 year [4]. ICH is more frequently seen in men than women, especially in the Japanese population, and ICH is twice as common in Asians compared to other ethnic groups. The incidence of ICH appears to increase with advanced age $[4,6]$.

\section{Pathophysiology}

ICH is classified as primary or secondary based on the underlying cause. Primary ICH is the result of spontaneous rupture of small vessels and accounts for 78 to $88 \%$ of all ICH cases [7]. Causes include hypertension and cerebral amyloid angiopathy. Secondary ICH (accounting for 12 to $22 \%$ of all ICH) is due to a cause other than small vessel rupture (e.g., aneurysm, arteriovenous malformation, hemorrhagic transformation of ischemic stroke, and neoplasms 
[7]. Following both types of ICH, edema formation will occur, perilesional blood flow will change, and some hematomas will expand with time. All these pathophysiological processes are described in more detail as follows, however, this review primarily focuses on the acute management of primary $\mathrm{ICH}$.

\section{Risk Factors}

Numerous risk factors for $\mathrm{ICH}$ have been identified over the past several decades, including genetics, race, lifestyle, and pre-existing medical conditions [7]. Genetic risk factors include the apolipoprotein E $\varepsilon 2$ and $\varepsilon 4$ alleles, whereas both raise the risk for ICH in the lobar brain regions, only the $\varepsilon 4$ allele is associated with deep ICH [8]. A first-degree relative with ICH is also an independent risk factor for ICH [9]. Lifestyle risk factors include smoking, excessive alcohol intake, drug abuse, unhealthy diet, and a lack of regular physical activity $[9,10]$. Risk factors in a patient's past medical history include prior stroke, hypertension, diabetes mellitus, psychosocial stress, cerebral amyloid angiopathy, coagulopathy, and an underlying vascular lesion [7, 9, 10]. Although many of these factors cannot be modified, some offer therapeutic targets. In particular, the population attributable risk of hypertension for ICH is quite high [9], and treatment of hypertension has been shown to decrease this risk for both cerebral amyloid angiopathy-related and hypertension-related ICH [11].

\section{Initial Hematoma}

Initial hematoma volume is the strongest predictor of 30 day mortality, with $96 \%$ sensitivity and $98 \%$ specificity [12]. In addition, it predicts poor functional outcome [13, 14]. The apolipoprotein $E \varepsilon 2$ allele is associated with larger hematomas, which appears to explain its effect on outcome [15]. The relation between oral anticoagulation use and baseline hematoma volume is unclear; some studies have shown increased baseline volumes [16, 17], whereas others have not $[15,18]$.

Hematoma location is also an important aspect of the initial hematoma, because location influences 30-day mortality rates: $44 \%$ for deep $\mathrm{ICH}, 46 \%$ for lobar $\mathrm{ICH}$, $60 \%$ for brainstem ICH, and 34\% for cerebellar ICH [3]. In a study of more than 1000 patients, the distribution of hematoma location showed 50\% deep, 35\% lobar, $10 \%$ cerebellar, and $6 \%$ brainstem ICH [19].

\section{Edema}

The presence of intracerebral hemorrhage causes edema formation surrounding the hematoma (termed perihemato- mal edema) starting within hours of ICH onset and progresses with time $[20,21]$. The physiology of edema formation consists of 2 stages. Early edema is due to the accumulation of serum proteins of the clot that contains osmotic activity [22]. Subsequently, the presence of cytotoxic and vasogenic edema leads to blood-brain barrier disturbances, sodium pump failure, and ultimately the death of neurons $[23,24]$. The first hours after ICH onset, the blood-brain barrier continues to be nonpermeable for larger molecules, but after 8 to 12 hours the permeability increases and therefore fosters further edema formation [20]. In addition, an inflammatory reaction starts early after $\mathrm{ICH}$ and peaks a few days post-ICH, leading to secondary brain injury [20].

\section{Perilesional Blood Flow}

In addition to edema, perilesional or perihematomal blood flow is of clinical interest because of a theoretical concern that lowering the systemic blood pressure may cause perilesional ischemia. Several studies, with heterogeneous results, have investigated this topic. A computed tomographic (CT) perfusion study showed reduced regional cerebral blood flow adjacent to the hematoma, which increased as the distance from the hematoma center increased [25]. In a single-photon emission computed tomography (SPECT) study, edema increased by $36 \%$ in the first 72 hours, and during this period the perilesional blood flow normalized [26]. An additional, more recent CT perfusion study also showed a reduced regional cerebral blood flow surrounding the hematoma, accompanied by a reduced oxygen extraction fraction. This decreased oxygen demand might be due to tissue damage caused by an inflammation process initiated by hematoma components [27]. Of note, 2 magnetic resonance image (MRI)-based studies found no evidence of decreased perilesional blood flow $[28,29]$.

\section{Hematoma Expansion}

Another predictor of poor outcome is ongoing bleeding after hospital arrival, or hematoma expansion. Seventy three percent of patients express some degree of expansion, and 30 to $40 \%$ of patients expand more than $33 \%$ from baseline volume [13, 18, 30, 31]. The pathophysiological mechanism by which hematoma expansion occurs remains unclear. Possibilities include ongoing bleeding from the initially ruptured vessel and a cascading injury of nearby damaged small vessels after the initial rupture of a vessel [32].

Hematoma expansion strongly influences outcome, with each $10 \%$ enlargement of the hematoma resulting in a $5 \%$ increased hazard of death, and an increase of $16 \%$ in the likelihood of 1 point worsening on the modified Rankin 
Scale [13]. Other studies also showed increased risks of poor functional outcome and death [16, 30, 33]. The occurrence of hematoma expansion is related to initial hematoma size; smaller hematomas are less likely to expand [16, 34, 35]. Other risk factors for expansion include early presentation [36], warfarin use [16, 18], and contrast extravasation on CT angiography (CTA) [36-38]. The latter will be discussed more extensively in the "Neuroimaging" section.

\section{Diagnosis}

\section{Clinical Signs}

Since ICH is a life-threatening disease, initial diagnosis is of critical importance. Clinical symptoms may include acute onset of headache, nausea, vomiting, seizures, and any focal or generalized neurological symptoms. In patients with suspected stroke, the presence of findings, such as coma, headache, vomiting, seizures, neck stiffness, and raised diastolic blood pressure, increase the likelihood of ICH compared to ischemia, but only neuroimaging can provide a definitive diagnosis [39].

\section{Neuroimaging}

\section{$C T$}

In the United States, CT is the most commonly used form of neuroimaging in patients with neurological deficits. CT scanners are rapidly and commonly available in emergency departments in the United States [40] and highly sensitive in detecting $\mathrm{ICH}$.

\section{CT Angiography}

$\mathrm{CT}$ angiography can accurately diagnose many underlying lesions of secondary $\mathrm{ICH}$, such as aneurysm, Moyamoya disease, neoplasms, and arteriovenous malformations [41]. In addition, CT venography is very accurate in diagnosing venous sinus thrombosis, another secondary cause of $\mathrm{ICH}$ [42]. As CT scanners are widely available [40], CT angiography is often used as an initial diagnostic modality in many centers, and may not meaningfully raise the risk of nephropathy following ICH [43].

Another advantage to CT angiography is its ability to predict hematoma expansion. In patients with primary $\mathrm{ICH}$, many show signs of contrast within the hematoma itself, suggesting that it may have been extravasated from an injured blood vessel $[36,44]$. The presence of this contrast, which has been termed a "spot sign," is an independent predictor of which patients will go on to suffer hematoma expansion (Fig. 1) [36-38]. The prevalence of the CTA spot sign decreases as time from onset to CTA increases, but its presence remains predictive of hematoma expansion independent of time from onset to CTA [36, 38]. Several other studies showed that the CTA spot sign is predictive of mortality and poor outcome $[45,46]$. In secondary ICH, the CTA spot sign is also predictive of in-hospital mortality [47], but vascular and nonvascular mimics of this finding are common and lower its sensitivity and specificity [48].

MRI

Although CT is the most commonly used diagnostic tool to detect ICH, gradient recalled echo MRI is equally sensitive to detect acute ICH [49]. In addition, it is more sensitive for small hemorrhages, termed "microbleeds" [50]. Although these may not cause acute symptoms, their presence can serve as a marker of underlying vascular disease and can risk stratify patients for recurrent ICH [51].
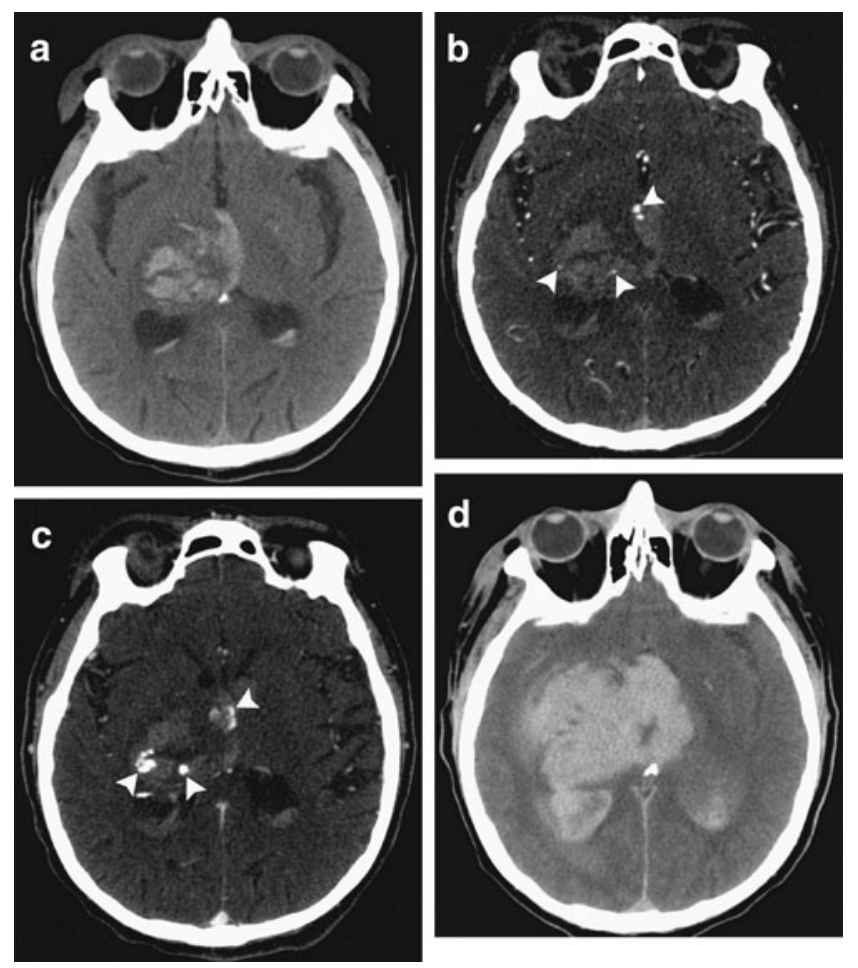

Fig. 1 Computed tomography (CT) and CT angiography of acute intracerebral hemorrhage. (A) Noncontrast CT shows a right thalamic intracerebral hemorrhage $(24 \mathrm{~mL})$ with associated intraventricular hemorrhage $(6 \mathrm{~mL})$. (B) CT angiography demonstrates 3 foci of contrast (spot signs) within the intracerebral hemorrhage (arrowheads). (C) Delayed CT angiography shows increased volume and changed morphology of the spot signs (arrowheads). (D) Noncontrast CT after 8 hours demonstrates expansion of the intracerebral hemorrhage $(94 \mathrm{~mL})$ and intraventricular hemorrhage $(82 \mathrm{~mL})$. The patient expired shortly after this follow-up CT. (Adapted from Delgado Almandoz JE, et al. Stroke 2009;40:2994-3000) 


\section{Management}

\section{General Principles}

Thus far, no individual therapy has demonstrated benefit in a randomized controlled trial. However, specialized treatment of acute $\mathrm{ICH}$ in a neurology or neurosurgery intensive care unit may well improve outcomes [52]. In addition, the fact that patients who have their care limited (such as those who have a "do not resuscitate" order) show worse outcomes, even in the absence of cardiac arrest, suggests that comprehensive aggressive care does provide some benefit [53]. Airway protection, anticoagulation reversal, blood pressure management, and intracranial pressure (ICP) management are vitally important strategies that need to be initiated as early as possible. In a recent article, 26 quality indicators were proposed for the systematic evaluation of $\mathrm{ICH}$ patient care, although many have not yet been validated as predictors of better outcome [54].

\section{Airway Protection}

Thirty percent of those with supratentorial $\mathrm{ICH}$ and the majority with cerebellar or brainstem $\mathrm{ICH}$ require intubation for airway protection [55]. Intubation can prevent aspiration and maximize oxygenation, but these benefits should be weighed against potential risks of intubation in the acute phase [56].

\section{Medical Management}

\section{Anticoagulation Reversal}

\section{Oral Anticoagulation}

A population-based study reported a fourfold increase in oral anticoagulation-associated ICH between 1988 and 1999, accounting for as much as $17 \%$ of ICH cases in 1999 [57]. The observed increase is most likely due to the increased use of warfarin by patients with atrial fibrillation [58]. The use of warfarin at the time of $\mathrm{ICH}$ is associated with hematoma expansion [16, 18] and increased mortality [15-17, 59].

Although no clinical trials have been published on anticoagulation reversal (the logistics of doing such a trial have been prohibitive [60]), the fact that anticoagulation predisposes to more bleeding suggests that anticoagulation reversal is probably beneficial. A rapid correction of the international normalized ratio (INR) is recommended by the American Heart Association (AHA) and the European Stroke Organization (ESO), formerly known as the European Stroke Initiative [61, 62]. Intravenous administration of vitamin $\mathrm{K}$ is recommended, but because its effect takes several hours, additional therapy to restore coagulation factors is recommended [61, 62]. Two products are thought to restore the vitamin K-dependent coagulation factors: fresh frozen plasma and four-factor prothrombin complex concentrates (PCC) (some PCCs are termed 3-factor as they contain minimal factor VII [63]). Correction of the INR to less than 1.4 within 2 hours has been associated with decreased rates of hematoma expansion [64], but it is difficult to achieve this with fresh frozen plasma alone [65]. The role of activated recombinant factor VIIa (rFVIIa) is unclear in patients with anticoagulation-associated $\mathrm{ICH}$, as restoring only 1 of the 4 missing factors may reverse the INR, but not necessarily the underlying coagulopathy $[66,67]$.

\section{Antiplatelet Therapy}

There have been conflicting studies as to whether antiplatelet use worsens outcome in $\mathrm{ICH}$; however, a recent systematic review suggested that previous use of antiplatelet therapy is associated with increased mortality [68]. This raises the question of whether antiplatelet therapy can be "reversed." The most commonly used intervention is transfusion of fresh platelets. However, the value of this is unclear and is therefore considered to be investigational by the AHA [61] and is currently under investigation in the Platelet Transfusion in Cerebral Haemorrhage (PATCH) trial (The Netherlands National Trial Register No. NTR1303) [69]. A future option might be pro-coagulant agents, such as rFVIIa [70]. However, for those patients with thrombocytopenia, platelet transfusion should be initiated [61].

\section{Direct Thrombin and Factor Xa Inhibitors}

Recent large clinical trials have suggested that oral factor $\mathrm{Xa}$ inhibitors (rivaroxaban and apixaban) [71, 72] and an oral direct thrombin inhibitor (dabigatran) [73] are effective alternatives to warfarin. Therefore, the near future holds the likelihood that $\mathrm{ICH}$ in the setting of these agents will become increasingly common. It is not at all clear how to "reverse" these agents; some authors have argued for coagulation factor concentrates, such as PCC [74, 75].

\section{Blood Pressure}

Elevated blood pressure is very common after ICH and is associated with hematoma expansion and poor outcome [76]. Unfortunately, it remains unclear whether elevated blood pressure is caused by expansion of the hematoma or if it is the cause of the expansion. Randomized controlled trials addressing acute blood pressure therapy include Intensive Blood Pressure Reduction in Acute Cerebral Haemorrhage Trial (INTERACT) [77] and Antihypertensive 
Treatment of Acute Cerebral Hemorrhage (ATACH) [78], and it appears that aggressive blood pressure treatment (to less than $140 \mathrm{mmHg}$ ) decreases the rate of substantial hematoma expansion without increasing adverse events [77, 78]. However, no effect on clinical outcome has been demonstrated. In a post-hoc analysis of the INTERACT trial, the maximum therapeutic effect on hematoma expansion was found in the group of patients with the lowest systolic blood pressure levels (median $135 \mathrm{mmHg}$ ) [79]. The INTERACT2 (ClinicalTrials.gov No. NCT00716079) and ATACH-II (ClinicalTrials.gov No. NCT01176565) trials are currently recruiting patients to further investigate whether blood pressure-lowering can improve outcomes.

\section{Recombinant Factor VIIa}

In 2 randomized clinical trials, the administration of rFVIIa to patients with primary ICH successfully reduced the risk of hematoma expansion, but did not change clinical outcomes $[80,81]$. This may have been due to an increased rate of thromboembolic events in the treatment arm [81]. Therefore, the AHA guidelines do not recommend the use of rFVIIa in unselected ICH patients. Two randomized placebo-controlled clinical trials, The Spot Sign for Predicting and Treating ICH Growth Study (STOP-IT) (ClinicalTrials.gov No. NCT00810888) and Spot Sign Selection of Intracerebral Hemorrhage to Guide Hemostatic Therapy (SPOTLIGHT) (ClinicalTrials.gov No. NCT01359202), are currently using the CTA spot sign to guide treatment with rFVIIa to select patients at their highest risk of hematoma expansion.

\section{Intracranial Pressure}

Elevated ICP is associated with neurological deterioration and poor outcome [82]. ICH patients with clinical or radiographic evidence of elevated ICP, therefore, may be candidates for interventions aimed at reducing it. ICP monitoring should be considered to guide therapy (see "ICP monitor placement"). Medical management aimed at reducing ICP includes hyperventilation and osmotic agents, which can improve cerebral blood flow and metabolism in animals with transtentorial herniation [83]. In humans, however, hyperventilation may be harmful outside of shortterm use while awaiting surgical decompression. Osmotic agents, such as mannitol and hypertonic saline, can be effective in the short-term management of transtentorial herniation or acute neurological deterioration associated with high intracranial pressure [84]. There is little data available on "desired" intracranial pressure ranges in ICH patients. The AHA guidelines, therefore, are partly based on data available from traumatic brain injury. The AHA recommends maintaining cerebral perfusion pressure between 50 and $70 \mathrm{mmHg}$, depending on cerebral autoregulation status
[61]. The ESO recommends a cerebral perfusion pressure greater than 60 to $70 \mathrm{mmHg}$ [62]. Because a randomized trial did not show any benefit of corticosteroids and showed higher complication rates in the treatment group, there is no clear role for them at this time [85].

\section{Supportive Care}

\section{Glucose}

A hyperglycemic state is common in the acute phase following ICH, affecting as much as $60 \%$ of patients. This independently predicts higher mortality rates in patients with and without diabetes [86, 87]. In addition, hyperglycemia is associated with larger hematoma volumes and more intraventricular hemorrhage (IVH) [86, 87]. The underlying pathophysiology of these findings remains unclear. Hypotheses include a nonspecific stress response or hypothalamic nuclei irritation [88]. In an experimental setting, a hyperglycemic state predisposes diabetic mice and rats to hematoma expansion compared to nondiabetic controls [89]. No randomized trial has been performed with only ICH patients, but 1 trial including critically ill patients in general showed improved functional outcomes with intensive management of glucose levels with insulin [90]. In contrast, the United Kingdom Glucose Insulin in Stroke Trial (GIST-UK) did not show any clinical benefit of intensive insulin therapy for a combined group of ICH and ischemic stroke patients, but the study was terminated early due to slow enrollment, and was therefore potentially underpowered to detect a significant difference between the 2 treatment groups [91]. Of note, all patients in these trials received therapy to correct hyperglycemia; the comparison was only subcutaneous versus intravenous insulin. No trial has included an arm in which hyperglycemia remains untreated, given the observational data on its harmful effects. Therefore, the AHA and ESO continue to recommend glucose monitoring and normoglycemia for all ICH patients in their most recent guidelines [61, 62].

\section{Temperature}

Fever is common in both deep and lobar ICH, especially in those with ventricular extension of their hematoma. After 3 days of hospital admission, fever and its duration are associated with poor clinical outcome [92]. This finding suggests that maintaining normothermia is favorable.

\section{Anemia}

Anemia is frequently found in ICH patients, even more so in those with larger hematomas [93]. Related to this finding, 
the transfusion of packed red blood cells is associated with increased 30-day survival rates [94]. However, both the AHA and ESO guidelines do not contain any specific advice on the treatment of anemia, and there is no clear target hemoglobin or hematocrit $[61,62]$.

\section{Surgical and Interventional Management}

\section{ICP Monitor Placement}

Monitoring of ICP is frequently performed in clinical practice, but is not extensively studied in the literature. Placement of an ICP monitor gives clinicians additional information with intubated or subconscious patients. In addition, an ICP monitor provides the opportunity to guide therapies aimed at reducing ICP and managing blood pressure while maximizing cerebral perfusion pressure [95]. Therefore, the AHA recommends considering the placement of an ICP monitor in patients with clinical signs of transtentorial herniation, a Glasgow Coma Scale (GCS) score of less than 9, hydrocephalus, or extensive IVH [61]. The ESO considers ICP monitor placement reasonable in mechanically ventilated patients [62]. An additional important treatment strategy consists of treating the underlying causes of elevated ICP, such as hydrocephalus or extensive intraventricular hemorrhage. As previously described, a cerebral perfusion pressure of approximately $70 \mathrm{mmHg}$ is considered to be reasonable.

\section{IVH and Hydrocephalus}

Intraventricular extension of ICH is relatively common, occurring in as much as $42 \%$ of patients [96]. Those with IVH suffer worse outcomes, and the amount of intraventricular blood relates directly to mortality [97]. A number of strategies are available to manage IVH. The most common is the placement of an external ventricular drain (EVD), which may reduce intracranial pressure; however, this effect is counterbalanced by the risk of infection and catheter obstruction by clots $[96,98]$. One group found that the intraventricular administration of thrombolytic agents to stimulate hemorrhage clearance may reduce mortality rates [99]; this has been followed up by the ongoing Clot Lysis Evaluating Accelerated Resolution of Intraventricular Hemorrhage III (CLEAR III) trial (ClinicalTrials.gov No. NCT00784134). Preliminary results show that the intraventricular administration of recombinant tissue-type plasminogen activator is safe; the symptomatic hemorrhage rate was $8 \%$ and bacterial ventriculitis was absent. The 30-day mortality rate of $8 \%$ was within the study safety limits [100].

As with IVH, hydrocephalus seems to predict poor outcome. In the International Surgical Trial in Intracerebral
Haemorrhage (STICH), 23\% of all ICH patients and 55\% of those with IVH had hydrocephalus. This was found to be a predictor of poor outcome [101]. Placement of an EVD for hydrocephalus relieves intracranial pressure and also provides ICP monitoring. Therefore, AHA and ESO guidelines consider EVD placement reasonable in selected patients with a decreased level of consciousness [61, 62].

\section{Surgical Clot Evacuation}

Surgical evacuation of hematomas remains controversial. Surgical evacuation reduces mass effect, and may minimize further damage and edema formation [102]. However, this effect is counterbalanced by the damage incurred during the approach through healthy tissue. This problem is most relevant for thalamic, basal ganglia, and pontine hemorrhages. The STICH trial [103] $(\mathrm{n}=1033)$, published in 2005, compared early surgery with medical treatment only. Patients were eligible for the study only if the responsible physician was uncertain as to the benefits of either treatment. At 6 months, early surgery (at a median time of $30 \mathrm{~h}$ from ICH onset) showed no benefit compared to conservative treatment; $24 \%$ versus $26 \%$ had good recovery or moderate disability. However, it may be that more easily accessed hematomas are a select group; in a subgroup analysis, early surgical treatment of lobar hematomas within $1 \mathrm{~cm}$ of the cortical surface were most likely to benefit [103]. The ongoing STICH II trial (International Standard Randomised Controlled Trial Number Register No. ISRCTN22153967) specifically tests the benefits of early surgery for lobar hematomas within $1 \mathrm{~cm}$ of the cortical surface without intraventricular extension [104]. One limitation of the original STICH trial was the timeframe to evacuation (median of $30 \mathrm{~h}$ ); it may be that any benefit of craniotomy is time dependent. However, a pilot study using the STICH trial protocol (except that patients were treated within 4 hours of symptom onset) showed higher rates of re-bleeds and poor outcomes [105]. Overall, for craniotomy in general, a Cochrane review of 10 trials pooling 2059 patients showed a statistically significant result in favor of surgery when looking at death as an endpoint alone, and also when death or dependency at final follow-up were combined [106].

\section{Cerebellar Hematomas}

A different subgroup of ICH consists of cerebellar hematomas. Several nonrandomized studies showed beneficial outcomes for cerebellar hematomas with a diameter exceeding $3 \mathrm{~cm}$, or causing hydrocephalus or brainstem compression, when surgically evacuated compared to medical treatment only [107-110]. In another study, best results were obtained by surgery in patients with a GCS 
below 14 or hematomas with a volume of $40 \mathrm{~mL}$ or more [111]. The AHA and ESO guidelines both recommend emergency surgery for cerebellar hematomas when patients are neurologically deteriorating or showing signs of brainstem compression and/or hydrocephalus [61, 62]. Initial treatment with an EVD instead of surgery is not recommended by the AHA [61].

\section{New Techniques}

New surgical techniques are emerging along the horizon of ICH treatment. Two promising techniques are stereotactic and endoscopic surgical evacuation of the hematoma. These approaches are believed to cause less damage to healthy tissue compared to an open craniotomy. A study of stereotactic-guided evacuation by endoscope showed a higher likelihood of good outcome compared to those who received the best medical care only [112]. A more recent study, the Stereotactic Treatment of Intracerebral Hematoma by Means of a Plasminogen Activator (SICHPA) trial, showed a significant volume reduction in the treatment arm of 10 to $20 \%$ within 72 hours of symptom onset. However, no significant effect was found on 6-month functional outcome or mortality [113]. In contrast, 2 other trials (one nonrandomized [114] and one randomized [115]) found stereotactic aspiration of deep $\mathrm{ICH}$ to be safe and associated with early improvement of National Institute of Health Stroke Scale (NIHSS) scores and improvement of long-term functional outcome measured by modified Rankin Scale [114, 115]. The ongoing Minimally Invasive Surgery plus T-PA for Intracerebral Hemorrhage Evacuation (MISTIE) clinical trial (ClinicalTrials.gov No. NCT00224770) investigates this therapeutic strategy in more depth by randomizing patients to either stereotactic aspiration with subsequent irrigation of the hematoma with thrombolytics or medical management only [104].

\section{Prevention of Complications}

\section{Seizures}

Seizures are a known complication of ICH. In patients undergoing continuous electroencephalographic monitoring, electrographic seizure frequency was $28 \%$ within $72 \mathrm{~h}$ after ICH. Most occurred in the first $24 \mathrm{~h}$, and these (mostly subclinical) seizures were seen more in lobar ICH patients and were associated with decreased NIHSS scores and increased midline shift. There was also a trend toward poorer functional outcome at discharge [116]. Treatment with antiepileptic drugs (AEDs) in lobar ICH patients is associated with a decrease in the number of clinically detectable seizures, which in theory should be beneficial [117]. However, AEDs have not been shown to improve outcome, and in fact may be associated with worse outcomes in unselected ICH patients [118, 119]. Therefore, the current AHA and ESO guidelines do not recommend the routine use of antiepileptic drugs in unselected $\mathrm{ICH}$ patients $[61,62]$. In a recent review, a treatment paradigm for the use of AEDs was proposed based on findings during neurological examination, ICH location, and the presence of clinical seizures [120].

\section{Deep Vein Thrombosis}

Deep vein thrombosis (DVT) is a common complication in hospitalized patients. Prevention of DVT is even more challenging in ICH patients given the concern that anticoagulation can increase the risk of bleeding. The incidence of asymptomatic DVT in ICH patients is approximately 10 to $16 \%[121,122]$. Stockings alone are not effective in preventing DVT in patients with ischemic or hemorrhagic strokes [122]. Intermittent pneumatic compression combined with elastic stockings is more effective than elastic stockings alone in preventing DVT in patients with ICH [121]. Therefore, the AHA and ESO recommendations state that patients with ICH should receive elastic stockings with additional pneumatic compression [61, 62].

In a study using a historical control group, early administration (day 2 after ICH) of subcutaneous lowdose heparin was associated with a decrease in pulmonary emboli without an increase in re-bleeding rates [123]. A recent retrospective study showed that the early use of subcutaneous low molecular weight heparin (LMWH) or unfractionated heparin does not lead to hematoma expansion in the acute (2-4 days) and subacute phase ( $\leq 7$ days) in both patients with ICH and patients with ICH and associated IVH [124]. Therefore, the AHA guidelines state that the administration of LMWH or unfractionated heparin may be considered after obtaining evidence of the discontinuance of bleeding [61]. The ESO guidelines consider LMWH to be safe after 24 hours from ICH onset [62].

\section{Prognostics}

\section{Outcome}

Despite technological advances, the overall mortality has not been significantly affected for the last several decades, and 1-month mortality remains approximately $40 \%$. In addition, after the first year, more than $75 \%$ of all patients are severely disabled or deceased $[1,4]$. Many patients, families, and providers wish to know, in the acute phase, what the patient's outcome will be ultimately. 
Outcome Prediction

Several predictors of outcome after ICH have been previously described and include age, NIHSS score, ICH location, baseline hematoma volume, anticoagulation use, and the presence and severity of IVH. Using these factors, several prediction models of ICH outcome have been developed. Two include the ICH score and FUNC score. The ICH score predicts 30-day mortality and includes age, GCS score, infratentorial location, IVH presence, and ICH volume (Table 1 and Fig. 2) [125]. The FUNC score predicts functional independence at 3 months and uses age, premedical history of cognitive impairment, GCS score, ICH location, and ICH volume (Fig. 3) [14].

\section{End-of-Life Decisions}

End-of-life decisions and the withdrawal of medical care are pivotally important in the outcome of ICH. A study including 1421 patients showed that withdrawal or limitations of care were the leading cause of death (68\%) in ICH patients [126]. Do not resuscitate orders have been shown to be an independent predictor of poor short- and long-term outcome after adjustment for ICH severity [53, 127]. It seems that the most important variable associated with outcome is the level of medical care provided. When providers take a more nihilistic approach, clinical outcomes are worse, even when a cardiac or respiratory arrest is absent [128]. Therefore,

Table 1 The ICH Score

\begin{tabular}{ll}
\hline Component & ICH Score Points \\
\hline GCS Score & \\
$3-4$ & 2 \\
$5-12$ & 1 \\
$13-15$ & 0 \\
ICH volume $\left(\mathrm{cm}^{3}\right)$ & \\
$\geq 30$ & 1 \\
$<30$ & 0 \\
IVH & \\
Yes & 1 \\
No & 0 \\
Infratentorial origin of ICH & \\
Yes & 1 \\
No & 0 \\
Age (years) & \\
$\geq 80$ & 1 \\
$<80$ & 0 \\
Total ICH score & $0-6$
\end{tabular}

GCS = Glasgow Coma Scale; ICH = intracerebral hemorrhage; IVH = intraventricular hemorrhage.

(Adapted from Hemphill JC, et al. Stroke 2001;32:891-897)

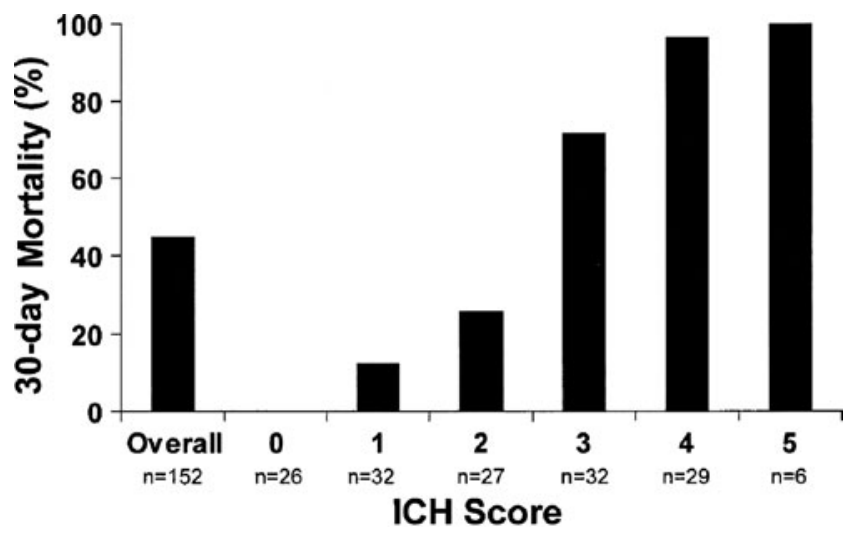

Fig. 2 The intracerebral hemorrhage (ICH) score and 30-day mortality are shown. The ICH score predicts 30-day mortality and includes age, Glasgow Coma Scale (GCS) score, infratentorial location, intraventricular hemorrhage (IVH) presence, and ICH volume. (Adapted from Hemphill JC, et al. Stroke 2001;32:891-897)

clinicians should be careful of adopting a "self fulfilling prophecy" in which selected patients receive less aggressive care overall, and the AHA in fact recommends deferring any care limitation order for the first $24 \mathrm{~h}$ after ICH [61].

\section{Prevention of Recurrent ICH}

The rate of recurrent hemorrhage is approximately $2 \%$ per patient-year [129, 130]. Several risk factors for recurrent ICH have been identified during the years, including older age [130], hypertension [129], lobar ICH location [131], possession of the apolipoprotein $\mathrm{E} \varepsilon 2$, or $\varepsilon 4$ allele [132], and more microbleeds on gradient recalled echo MRI [51]. Of these, hypertension is an important and modifiable risk factor for recurrent hemorrhage. In patients with a diastolic blood pressure greater than $90 \mathrm{mmHg}$, the recurrence rate of $\mathrm{ICH}$ is $10 \%$ per patient-year compared to $1.5 \%$ in patients with a diastolic blood pressure below $90 \mathrm{mmHg}$

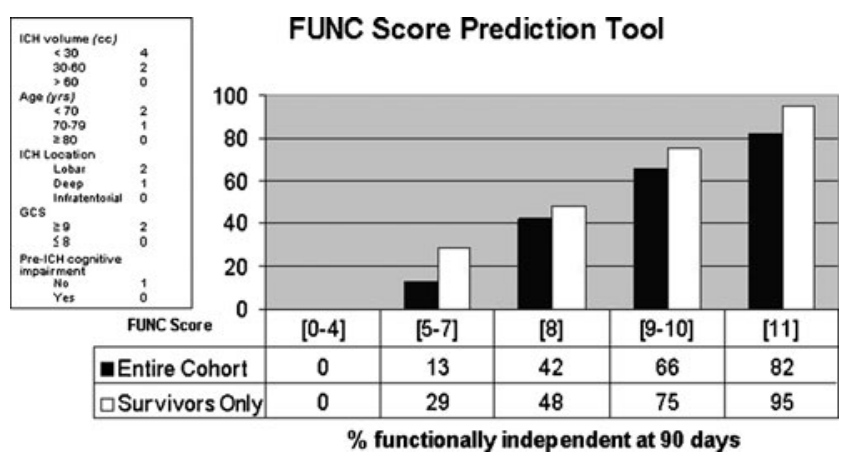

Fig. 3 The FUNC score predicts functional independence at 3 months and uses age, premedical history of cognitive impairment, GCS score, ICH location and ICH volume. (Adapted from Rost N.S., et al. Stroke 2008;39:2304-2309) 
[129]. In the previously described Perindopril Protection against Recurrent Stroke Study (PROGRESS) trial, blood pressure lowering was also associated with a reduced risk of recurrent ICH [11]. Therefore, appropriate blood pressure regulation, before and after $\mathrm{ICH}$, is a pivotal treatment aim for physicians involved in the care of ICH patients.

In an observational study, antiplatelet therapy after ICH was common and seemed not to be associated with the recurrence of both deep and lobar ICH [131]. However, oral anticoagulation use after ICH is associated with a threefold increase in recurrent ICH [130]. It is likely that some patients can be safely re-anticoagulated (such as those with hypertensive ICH in whom good blood pressure control can be achieved), but some cannot, and the appropriate timeframe is likely a function of their risk [133]. Recent studies have highlighted a role for oral thrombin and factor Xa inhibitors as warfarin alternatives [71-73].

\section{Future Directions}

Many clinical trials are planned or actively enrolling patients, and the near future may hold a wide range of new therapies. To minimize the risk of hematoma expansion, investigators are testing platelet transfusion (PATCH trial), anticoagulation reversal (INCH [ClinicalTrials.gov No. NCT00928915] and Beriplex [ClinicalTrials.gov No. NCT00708435] trials), blood pressure-lowering (INTERACT2 and ATACH-II), and hemostatic therapy guided by CTA findings (STOP-IT and SPOTLIGHT). To minimize mass effect of the hematoma, the STICH II trial selects patients with lobar hematomas within $1 \mathrm{~cm}$ of the cortical surface for hematoma evacuation, and the MISTIE trial is evaluating minimally invasive surgical techniques. The clearance of intraventricular blood with thrombolytics is the subject of the CLEAR III trial. Finally, novel potential neuroprotective agents are under investigation. One of the recently published agents (deferoxamine) may help minimize neuronal injury following ICH, and a phase II trial is under consideration [134]. Other neuroprotective agents studied in ongoing trials include statins (Simvastatin for Intracerebral Hemorrhage Study; ClinicalTrials.gov No. NCT00718328), albumin (ACHIEVE; ClinicalTrials.gov No. NCT00990509), celecoxib (ACE-ICH; ClinicalTrials. gov No. NCT00526214), and pioglitazone (SHRINC; ClinicalTrials.gov No. NCT00827892). In conclusion, the next several years may see numerous advances in the care of patients with ICH as a result of these ongoing studies.

Acknowledgments All funding entities had no involvement in study design, data collection and interpretation, writing of the manuscript and decision to submit it for publication. The study was funded by the National Institutes of Health $(\mathrm{NIH})$ - National Institute of Neurological Disorders and Stroke (NINDS) grant No. 5K23NS059774. Dr.
Brouwers was supported by the NIH - NINDS Specialized Program of Translational Research in Acute Stroke (SPOTRIAS) fellowship grant No. P50NS051343.

Required Author Forms Disclosure forms provided by the authors are available with the online version of this article.

Disclosures H.B. Brouwers has no disclosures; J.N. Goldstein discloses research grants from NINDS, and work as a consultant and on advisory boards for CSL Behring. Full conflict of interest disclosures is available in the electronic supplementary material for this article.

\section{References}

1. Qureshi AI, Mendelow AD, Hanley DF. Intracerebral haemorrhage. Lancet 2009;373:1632-1644.

2. Dennis MS. Outcome after brain haemorrhage. Cerebrovasc Dis 2003;16(suppl 1:9-13.

3. Flaherty ML, Haverbusch M, Sekar P, et al. Long-term mortality after intracerebral hemorrhage. Neurology 2006;66:1182-1186.

4. van Asch CJ, Luitse MJ, Rinkel GJ, van der Tweel I, Algra A, Klijn CJ. Incidence, case fatality, and functional outcome of intracerebral haemorrhage over time, according to age, sex, and ethnic origin: a systematic review and meta-analysis. Lancet Neurol 2010;9:167-176.

5. Kleindorfer DO, Khoury J, Moomaw CJ, et al. Stroke incidence is decreasing in whites but not in blacks: a population-based estimate of temporal trends in stroke incidence from the Greater Cincinnati/ Northern Kentucky Stroke Study. Stroke 2010;41:1326-1331.

6. Broderick JP, Brott T, Tomsick T, Huster G, Miller R. The risk of subarachnoid and intracerebral hemorrhages in blacks as compared with whites. N Engl J Med 1992;326:733-736.

7. Qureshi AI, Tuhrim S, Broderick JP, Batjer HH, Hondo H, Hanley DF. Spontaneous intracerebral hemorrhage. N Engl J Med 2001;344:1450-1460.

8. Biffi A, Sonni A, Anderson CD, et al. Variants at APOE influence risk of deep and lobar intracerebral hemorrhage. Ann Neurol 2010;68:934-943.

9. Woo D, Sauerbeck LR, Kissela BM, et al. Genetic and environmental risk factors for intracerebral hemorrhage: preliminary results of a population-based study. Stroke 2002;33:1190-1195.

10. O'Donnell MJ, Xavier D, Liu L, et al. Risk factors for ischaemic and intracerebral haemorrhagic stroke in 22 countries (the INTERSTROKE study): a case-control study. Lancet 2010;376:112-123.

11. Arima H, Tzourio C, Anderson C, et al. Effects of perindoprilbased lowering of blood pressure on intracerebral hemorrhage related to amyloid angiopathy: the PROGRESS trial. Stroke 2010;41:394-396.

12. Broderick JP, Brott TG, Duldner JE, Tomsick T, Huster G. Volume of intracerebral hemorrhage. A powerful and easy-to-use predictor of 30-day mortality. Stroke 1993;24:987-993.

13. Davis SM, Broderick J, Hennerici M, et al. Hematoma growth is a determinant of mortality and poor outcome after intracerebral hemorrhage. Neurology 2006;66:1175-1181.

14. Rost NS, Smith EE, Chang Y, et al. Prediction of functional outcome in patients with primary intracerebral hemorrhage: the FUNC score. Stroke 2008;39:2304-2309.

15. Biffi A, Anderson CD, Jagiella JM, et al. APOE genotype and extent of bleeding and outcome in lobar intracerebral haemorrhage: a genetic association study. Lancet Neurol 2011;10:702-709.

16. Cucchiara B, Messe S, Sansing L, Kasner S, Lyden P, CHANT Investigators. Hematoma growth in oral anticoagulant related intracerebral hemorrhage. Stroke 2008;39:2993-2996. 
17. Flaherty ML, Tao H, Haverbusch M, et al. Warfarin use leads to larger intracerebral hematomas. Neurology 2008;71:1084-1089.

18. Flibotte JJ, Hagan N, O'Donnell J, Greenberg SM, Rosand J. Warfarin, hematoma expansion, and outcome of intracerebral hemorrhage. Neurology 2004;63:1059-1064.

19. Flaherty ML, Woo D, Haverbusch M, et al. Racial variations in location and risk of intracerebral hemorrhage. Stroke 2005;36:934-937.

20. Xi G, Keep RF, Hoff JT. Mechanisms of brain injury after intracerebral haemorrhage. Lancet Neurol 2006;5:53-63.

21. Staykov D, Wagner I, Volbers B, et al. Natural course of perihemorrhagic edema after intracerebral hemorrhage. Stroke 2011;42:2625-2629.

22. Wagner KR, Xi G, Hua Y, et al. Lobar intracerebral hemorrhage model in pigs: rapid edema development in perihematomal white matter. Stroke 1996;27:490-497.

23. Mun-Bryce S, Kroh FO, White J, Rosenberg GA. Brain lactate and $\mathrm{pH}$ dissociation in edema: $1 \mathrm{H}-$ and $31 \mathrm{P}-\mathrm{NMR}$ in collagenase-induced hemorrhage in rats. Am $\mathrm{J}$ Physiol 1993;265:R697-702.

24. Wagner KR, Xi G, Hua Y, Kleinholz M, de Courten-Myers GM, Myers RE. Early metabolic alterations in edematous perihematomal brain regions following experimental intracerebral hemorrhage. J Neurosurg 1998;88:1058-1065.

25. Rosand J, Eskey C, Chang Y, Gonzalez RG, Greenberg SM, Koroshetz WJ. Dynamic single-section CT demonstrates reduced cerebral blood flow in acute intracerebral hemorrhage. Cerebrovasc Dis 2002;14:214-220.

26. Mayer SA, Lignelli A, Fink ME, et al. Perilesional blood flow and edema formation in acute intracerebral hemorrhage: a SPECT study. Stroke 1998;29:1791-1798.

27. Herweh C, Juttler E, Schellinger PD, et al. Evidence against a perihemorrhagic penumbra provided by perfusion computed tomography. Stroke 2007;38:2941-2947.

28. Kidwell CS, Saver JL, Mattiello J, et al. Diffusion-perfusion MR evaluation of perihematomal injury in hyperacute intracerebral hemorrhage. Neurology 2001;57:1611-1617.

29. Schellinger PD, Fiebach JB, Hoffmann K, et al. Stroke MRI in intracerebral hemorrhage: is there a perihemorrhagic penumbra? Stroke 2003;34:1674-1679.

30. Brott T, Broderick J, Kothari R, et al. Early hemorrhage growth in patients with intracerebral hemorrhage. Stroke 1997;28:1-5.

31. Mayer SA. Ultra-early hemostatic therapy for intracerebral hemorrhage. Stroke 2003;34:224-229.

32. Fisher CM. Pathological observations in hypertensive cerebral hemorrhage. J Neuropathol Exp Neurol 1971;30:536-550.

33. Dowlatshahi D, Demchuk AM, Flaherty ML, et al. Defining hematoma expansion in intracerebral hemorrhage: relationship with patient outcomes. Neurology 2011;76:1238-1244.

34. Broderick JP, Diringer MN, Hill MD, et al. Determinants of intracerebral hemorrhage growth: an exploratory analysis. Stroke 2007;38:1072-1075.

35. Dowlatshahi D, Smith EE, Flaherty ML, et al. Small intracerebral haemorrhages are associated with less haematoma expansion and better outcomes. Int J Stroke 2011;6:201-206.

36. Goldstein JN, Fazen LE, Snider R, et al. Contrast extravasation on $\mathrm{CT}$ angiography predicts hematoma expansion in intracerebral hemorrhage. Neurology 2007;68:889-894.

37. Wada R, Aviv RI, Fox AJ, et al. CT angiography "spot sign" predicts hematoma expansion in acute intracerebral hemorrhage. Stroke 2007;38:1257-1262.

38. Delgado Almandoz JE, Yoo AJ, Stone MJ, et al. Systematic characterization of the computed tomography angiography spot sign in primary intracerebral hemorrhage identifies patients at highest risk for hematoma expansion: the spot sign score. Stroke 2009;40:2994-3000.
39. Runchey S, McGee S. Does this patient have a hemorrhagic stroke? Clinical findings distinguishing hemorrhagic stroke from ischemic stroke. JAMA 2010;303:2280-2286.

40. Ginde AA, Foianini A, Renner DM, Valley M, Camargo CA Jr. Availability and quality of computed tomography and magnetic resonance imaging equipment in U.S. emergency departments. Acad Emerg Med 2008;15:780-783.

41. Delgado Almandoz JE, Romero JM. Advanced CT imaging in the evaluation of hemorrhagic stroke. Neuroimaging Clin N Am 2011;21:197-213.

42. Linn J, Ertl-Wagner B, Seelos KC, et al. Diagnostic value of multidetector-row CT angiography in the evaluation of thrombosis of the cerebral venous sinuses. AJNR Am J Neuroradiol 2007;28:946-952.

43. Oleinik A, Romero JM, Schwab K, et al. CT angiography for intracerebral hemorrhage does not increase risk of acute nephropathy. Stroke 2009;40:2393-2397.

44. Becker KJ, Baxter AB, Bybee HM, Tirschwell DL, Abouelsaad $\mathrm{T}$, Cohen WA. Extravasation of radiographic contrast is an independent predictor of death in primary intracerebral hemorrhage. Stroke 1999;30:2025-2032.

45. Kim J, Smith A, Hemphill JC 3rd, et al. Contrast extravasation on $\mathrm{CT}$ predicts mortality in primary intracerebral hemorrhage. AJNR Am J Neuroradiol 2008;29:520-525.

46. Delgado Almandoz JE, Yoo AJ, Stone MJ, et al. The spot sign score in primary intracerebral hemorrhage identifies patients at highest risk of in-hospital mortality and poor outcome among survivors. Stroke 2010;41:54-60.

47. Delgado Almandoz JE, Kelly HR, Schaefer PW, et al. CT angiography spot sign predicts in-hospital mortality in patients with secondary intracerebral hemorrhage. J Neurointerv Surg 2011 Oct 15. [Epub ahead of print]

48. Gazzola S, Aviv RI, Gladstone DJ, et al. Vascular and nonvascular mimics of the CT angiography "spot sign" in patients with secondary intracerebral hemorrhage. Stroke 2008;39:1177-1183.

49. Kidwell CS, Chalela JA, Saver JL, et al. Comparison of MRI and $\mathrm{CT}$ for detection of acute intracerebral hemorrhage. JAMA 2004;292:1823-1830.

50. Greenberg SM, Vernooij MW, Cordonnier C, et al. Cerebral microbleeds: a guide to detection and interpretation. Lancet Neurol 2009;8:165-174.

51. Greenberg SM, Eng JA, Ning M, Smith EE, Rosand J. Hemorrhage burden predicts recurrent intracerebral hemorrhage after lobar hemorrhage. Stroke 2004;35:1415-1420.

52. Diringer MN, Edwards DF. Admission to a neurologic/neurosurgical intensive care unit is associated with reduced mortality rate after intracerebral hemorrhage. Crit Care Med 2001;29:635-640.

53. Hemphill JC 3rd, Newman J, Zhao S, Johnston SC. Hospital usage of early do-not-resuscitate orders and outcome after intracerebral hemorrhage. Stroke 2004;35:1130-1134.

54. Qureshi AI. Intracerebral hemorrhage specific intensity of care quality metrics. Neurocrit Care 2011;14:291-317.

55. Gujjar AR, Deibert E, Manno EM, Duff S, Diringer MN. Mechanical ventilation for ischemic stroke and intracerebral hemorrhage: indications, timing, and outcome. Neurology 1998;51:447-451.

56. Lecky F, Bryden D, Little R, Tong N, Moulton C. Emergency intubation for acutely ill and injured patients. Cochrane Database Syst Rev 2008;2:CD001429.

57. Flaherty ML, Kissela B, Woo D, et al. The increasing incidence of anticoagulant-associated intracerebral hemorrhage. Neurology 2007;68:116-121.

58. Steiner T, Rosand J, Diringer M. Intracerebral hemorrhage associated with oral anticoagulant therapy: current practices and unresolved questions. Stroke 2006;37:256-262.

59. Rosand J, Eckman MH, Knudsen KA, Singer DE, Greenberg SM. The effect of warfarin and intensity of anticoagulation on 
outcome of intracerebral hemorrhage. Arch Intern Med 2004; $164: 880-884$.

60. Flaherty ML, Adeoye O, Sekar P, et al. The challenge of designing a treatment trial for warfarin-associated intracerebral hemorrhage. Stroke 2009;40:1738-1742.

61. Morgenstern LB, Hemphill JC 3rd, Anderson C, et al. Guidelines for the management of spontaneous intracerebral hemorrhage: a guideline for healthcare professionals from the American Heart Association/American Stroke Association. Stroke 2010;41:2108-2129.

62. European Stroke Initiative Writing Committee, Writing Committee for the EUSI Executive Committee, Steiner T, et al. Recommendations for the management of intracranial haemorrhage - part I: spontaneous intracerebral haemorrhage. The European Stroke Initiative Writing Committee and the Writing Committee for the EUSI Executive Committee. Cerebrovasc Dis 2006;22:294-316.

63. Holland L, Warkentin TE, Refaai M, Crowther MA, Johnston MA, Sarode R. Suboptimal effect of a three-factor prothrombin complex concentrate (Profilnine-SD) in correcting supratherapeutic international normalized ratio due to warfarin overdose. Transfusion 2009;49:1171-1177.

64. Huttner HB, Schellinger PD, Hartmann M, et al. Hematoma growth and outcome in treated neurocritical care patients with intracerebral hemorrhage related to oral anticoagulant therapy: comparison of acute treatment strategies using vitamin $\mathrm{K}$, fresh frozen plasma, and prothrombin complex concentrates. Stroke 2006;37:1465-1470.

65. Goldstein JN, Rosand J, Schwamm LH. Warfarin reversal in anticoagulant-associated intracerebral hemorrhage. Neurocrit Care 2008;9:277-283.

66. Tanaka KA, Szlam F, Dickneite G, Levy JH. Effects of prothrombin complex concentrate and recombinant activated factor VII on vitamin $\mathrm{K}$ antagonist induced anticoagulation. Thromb Res 2008;122:117-123.

67. Skolnick BE, Mathews DR, Khutoryansky NM, Pusateri AE, Carr ME. Exploratory study on the reversal of warfarin with rFVIIa in healthy subjects. Blood 2010;116:693-701.

68. Thompson BB, Bejot Y, Caso V, et al. Prior antiplatelet therapy and outcome following intracerebral hemorrhage: a systematic review. Neurology 2010;75:1333-1342.

69. de Gans K, de Haan RJ, Majoie CB, et al. PATCH: platelet transfusion in cerebral haemorrhage: study protocol for a multicentre, randomised, controlled trial. BMC Neurol 2010;10:19.

70. Skolnick BE, Shenouda M, Khutoryansky NM, Pusateri AE, Gabriel D, Carr ME. Reversal of clopidogrel-induced bleeding with rFVIIa in healthy subjects: a randomized, placebocontrolled, double-blind, exploratory study. Anesth Analg 2011;113:703-710.

71. Granger CB, Alexander JH, McMurray JJ, et al. Apixaban versus Warfarin in patients with atrial fibrillation. $N$ Engl J Med 2011;365:981-992.

72. Patel MR, Mahaffey KW, Garg J, et al. Rivaroxaban versus warfarin in nonvalvular atrial fibrillation. $N$ Engl J Med 2011;365:883-891.

73. Connolly SJ, Ezekowitz MD, Yusuf S, et al. Dabigatran versus warfarin in patients with atrial fibrillation. $N$ Engl J Med 2009;361:1139-1151.

74. van Ryn J, Stangier J, Haertter S, et al. Dabigatran etexilate - a novel, reversible, oral direct thrombin inhibitor: interpretation of coagulation assays and reversal of anticoagulant activity. Thromb Haemost 2010;103:1116-1127.

75. Eerenberg ES, Kamphuisen PW, Sijpkens MK, Meijers JC, Buller HR, Levi M. Reversal of Rivaroxaban and Dabigatran by prothrombin complex concentrate: a randomized, placebo-controlled, crossover study in healthy subjects. Circulation 2011;124:1573-1579.

76. Carlberg B, Asplund K, Hagg E. The prognostic value of admission blood pressure in patients with acute stroke. Stroke 1993;24:1372-1375.
77. Anderson CS, Huang Y, Wang JG, et al. Intensive blood pressure reduction in acute cerebral haemorrhage trial (INTERACT): a randomised pilot trial. Lancet Neurol 2008;7:391-399.

78. Antihypertensive Treatment of Acute Cerebral Hemorrhage (ATACH) investigators. Antihypertensive treatment of acute cerebral hemorrhage. Crit Care Med 2010;38:637-648.

79. Arima H, Anderson CS, Wang JG, et al. Lower treatment blood pressure is associated with greatest reduction in hematoma growth after acute intracerebral hemorrhage. Hypertension 2010;56:852-858.

80. Mayer SA, Brun NC, Begtrup K, et al. Recombinant activated factor VII for acute intracerebral hemorrhage. N Engl J Med 2005;352:777-785.

81. Mayer SA, Brun NC, Begtrup K, et al. Efficacy and safety of recombinant activated factor VII for acute intracerebral hemorrhage. N Engl J Med 2008;358:2127-2137.

82. Fernandes HM, Siddique S, Banister K, et al. Continuous monitoring of ICP and CPP following ICH and its relationship to clinical, radiological and surgical parameters. Acta Neurochir Suppl 2000;76:463-466.

83. Qureshi AI, Wilson DA, Traystman RJ. Treatment of elevated intracranial pressure in experimental intracerebral hemorrhage: comparison between mannitol and hypertonic saline. Neurosurgery 1999;44:1055-1063.

84. Misra UK, Kalita J, Ranjan P, Mandal SK. Mannitol in intracerebral hemorrhage: a randomized controlled study. J Neurol Sci 2005;234:41-45.

85. Poungvarin N, Bhoopat W, Viriyavejakul A, et al. Effects of dexamethasone in primary supratentorial intracerebral hemorrhage. N Engl J Med 1987;316:1229-1233.

86. Passero S, Ciacci G, Ulivelli M. The influence of diabetes and hyperglycemia on clinical course after intracerebral hemorrhage. Neurology 2003;61:1351-1356.

87. Stead LG, Jain A, Bellolio MF, et al. Emergency Department hyperglycemia as a predictor of early mortality and worse functional outcome after intracerebral hemorrhage. Neurocrit Care 2010;13:67-74.

88. Appelboom G, Piazza MA, Hwang BY, et al. Severity of intraventricular extension correlates with level of admission glucose after intracerebral hemorrhage. Stroke 2011;42:18831888.

89. Liu J, Gao BB, Clermont AC, et al. Hyperglycemia-induced cerebral hematoma expansion is mediated by plasma kallikrein. Nat Med 2011;17:206-210.

90. van den Berghe G, Wouters P, Weekers F, et al. Intensive insulin therapy in the critically ill patients. $N$ Engl J Med 2001;345:1359-1367.

91. Gray CS, Hildreth AJ, Sandercock PA, et al. Glucose-potassiuminsulin infusions in the management of post-stroke hyperglycaemia: the UK Glucose Insulin in Stroke Trial (GIST-UK). Lancet Neurol 2007;6:397-406.

92. Schwarz S, Hafner K, Aschoff A, Schwab S. Incidence and prognostic significance of fever following intracerebral hemorrhage. Neurology 2000;54:354-361.

93. Kumar MA, Rost NS, Snider RW, et al. Anemia and hematoma volume in acute intracerebral hemorrhage. Crit Care Med 2009;37:1442-1447.

94. Sheth KN, Gilson AJ, Chang Y, et al. Packed red blood cell transfusion and decreased mortality in intracerebral hemorrhage. Neurosurgery 2011;68:1286-1292.

95. Lavinio A, Menon DK. Intracranial pressure: why we monitor it, how to monitor it, what to do with the number and what's the future? Curr Opin Anaesthesiol 2011;24:117-123.

96. Hanley DF. Intraventricular hemorrhage: severity factor and treatment target in spontaneous intracerebral hemorrhage. Stroke 2009;40:1533-1538. 
97. Tuhrim S, Horowitz DR, Sacher M, Godbold JH. Volume of ventricular blood is an important determinant of outcome in supratentorial intracerebral hemorrhage. Crit Care Med 1999;27:617-621.

98. Adams RE, Diringer MN. Response to external ventricular drainage in spontaneous intracerebral hemorrhage with hydrocephalus. Neurology 1998;50:519-523.

99. Naff NJ, Carhuapoma JR, Williams MA, et al. Treatment of intraventricular hemorrhage with urokinase: effects on 30-day survival. Stroke 2000;31:841-847.

100. Morgan T, Awad I, Keyl P, Lane K, Hanley D. Preliminary report of the clot lysis evaluating accelerated resolution of intraventricular hemorrhage (CLEAR-IVH) clinical trial. Acta Neurochir Suppl 2008;105:217-220.

101. Bhattathiri PS, Gregson B, Prasad KS, Mendelow AD, STICH Investigators. Intraventricular hemorrhage and hydrocephalus after spontaneous intracerebral hemorrhage: results from the STICH trial. Acta Neurochir Suppl 2006;96:65-68.

102. Kaufman HH. Treatment of deep spontaneous intracerebral hematomas. A review. Stroke 1993;24:I101-106.

103. Mendelow AD, Gregson BA, Fernandes HM, et al. Early surgery versus initial conservative treatment in patients with spontaneous supratentorial intracerebral haematomas in the International Surgical Trial in Intracerebral Haemorrhage (STICH): a randomised trial. Lancet 2005;365:387-397.

104. Mendelow AD, Unterberg A. Surgical treatment of intracerebral haemorrhage. Curr Opin Crit Care 2007;13:169-174.

105. Morgenstern LB, Demchuk AM, Kim DH, Frankowski RF, Grotta JC. Rebleeding leads to poor outcome in ultra-early craniotomy for intracerebral hemorrhage. Neurology 2001;56:1294-1299.

106. Prasad K, Mendelow AD, Gregson B. Surgery for primary supratentorial intracerebral haemorrhage. Cochrane Database Syst Rev 2008;4:CD000200.

107. Da Pian R, Bazzan A, Pasqualin A. Surgical versus medical treatment of spontaneous posterior fossa haematomas: a cooperative study on 205 cases. Neurol Res 1984;6:145-151.

108. van Loon J, Van Calenbergh F, Goffin J, Plets C. Controversies in the management of spontaneous cerebellar haemorrhage. A consecutive series of 49 cases and review of the literature. Acta Neurochir (Wien) 1993;122:187-193.

109. Kirollos RW, Tyagi AK, Ross SA, van Hille PT, Marks PV. Management of spontaneous cerebellar hematomas: a prospective treatment protocol. Neurosurgery 2001;49:1378-1386.

110. Morioka J, Fujii M, Kato S, et al. Surgery for spontaneous intracerebral hemorrhage has greater remedial value than conservative therapy. Surg Neurol 2006;65:67-72.

111. Kobayashi S, Sato A, Kageyama Y, Nakamura H, Watanabe Y, Yamaura A. Treatment of hypertensive cerebellar hemorrhagesurgical or conservative management? Neurosurgery 1994;34:246-250.

112. Auer LM, Deinsberger W, Niederkorn K, et al. Endoscopic surgery versus medical treatment for spontaneous intracerebral hematoma: a randomized study. J Neurosurg 1989;70:530535.

113. Teernstra OP, Evers SM, Lodder J, et al. Stereotactic treatment of intracerebral hematoma by means of a plasminogen activator: a multicenter randomized controlled trial (SICHPA). Stroke 2003;34:968-974.

114. Vespa P, McArthur D, Miller C, et al. Frameless stereotactic aspiration and thrombolysis of deep intracerebral hemorrhage is associated with reduction of hemorrhage volume and neurological improvement. Neurocrit Care 2005;2:274-281.
115. Wang WZ, Jiang B, Liu HM, et al. Minimally invasive craniopuncture therapy vs. conservative treatment for spontaneous intracerebral hemorrhage: results from a randomized clinical trial in China. Int J Stroke 2009;4:11-16.

116. Vespa PM, O'Phelan K, Shah M, et al. Acute seizures after intracerebral hemorrhage: a factor in progressive midline shift and outcome. Neurology 2003;60:1441-1446.

117. Passero S, Rocchi R, Rossi S, Ulivelli M, Vatti G. Seizures after spontaneous supratentorial intracerebral hemorrhage. Epilepsia 2002;43:1175-1180.

118. Messe SR, Sansing LH, Cucchiara BL, et al. Prophylactic antiepileptic drug use is associated with poor outcome following ICH. Neurocrit Care 2009;11:38-44.

119. Naidech AM, Garg RK, Liebling S, et al. Anticonvulsant use and outcomes after intracerebral hemorrhage. Stroke 2009;40:3810-3815.

120. Gilmore E, Choi HA, Hirsch LJ, Claassen J. Seizures and CNS hemorrhage: spontaneous intracerebral and aneurysmal subarachnoid hemorrhage. Neurologist 2010;16:165-175.

121. Lacut K, Bressollette L, Le Gal G, et al. Prevention of venous thrombosis in patients with acute intracerebral hemorrhage. Neurology 2005;65:865-869.

122. CLOTS Trials Collaboration, Dennis M, Sandercock PA, et al. Effectiveness of thigh-length graduated compression stockings to reduce the risk of deep vein thrombosis after stroke (CLOTS trial 1): a multicentre, randomised controlled trial. Lancet 2009;373:1958-1965.

123. Boeer A, Voth E, Henze T, Prange HW. Early heparin therapy in patients with spontaneous intracerebral haemorrhage. J Neurol Neurosurg Psychiatry 1991;54:466-467.

124. Wu TC, Kasam M, Harun N, et al. Pharmacological deep vein thrombosis prophylaxis does not lead to hematoma expansion in intracerebral hemorrhage with intraventricular extension. Stroke 2011;42:705-709.

125. Hemphill JC 3rd, Bonovich DC, Besmertis L, Manley GT, Johnston SC. The ICH score: a simple, reliable grading scale for intracerebral hemorrhage. Stroke 2001;32:891-897.

126. Zurasky JA, Aiyagari V, Zazulia AR, Shackelford A, Diringer MN. Early mortality following spontaneous intracerebral hemorrhage. Neurology 2005;64:725-727.

127. Zahuranec DB, Brown DL, Lisabeth LD, et al. Early care limitations independently predict mortality after intracerebral hemorrhage. Neurology 2007;68:1651-1657.

128. Becker KJ, Baxter AB, Cohen WA, et al. Withdrawal of support in intracerebral hemorrhage may lead to self-fulfilling prophecies. Neurology 2001;56:766-772.

129. Arakawa S, Saku Y, Ibayashi S, Nagao T, Fujishima M. Blood pressure control and recurrence of hypertensive brain hemorrhage. Stroke 1998;29:1806-1809.

130. Vermeer SE, Algra A, Franke CL, Koudstaal PJ, Rinkel GJ. Long-term prognosis after recovery from primary intracerebral hemorrhage. Neurology 2002;59:205-209.

131. Viswanathan A, Rakich SM, Engel C, et al. Antiplatelet use after intracerebral hemorrhage. Neurology 2006;66:206-209.

132. O'Donnell HC, Rosand J, Knudsen KA, et al. Apolipoprotein E genotype and the risk of recurrent lobar intracerebral hemorrhage. N Engl J Med 2000;342:240-245.

133. Goldstein JN, Greenberg SM. Should anticoagulation be resumed after intracerebral hemorrhage? Cleve Clin J Med 2010;77:791-799.

134. Selim M, Yeatts S, Goldstein JN, et al. Safety and tolerability of deferoxamine mesylate in patients with acute intracerebral hemorrhage. Stroke 2011;42:3067-3074. 